\title{
Interaction Between Smooth Anticipation and Saccades During Ocular Orientation in Darkness
}

\author{
GUNNAR BLOHM, ${ }^{1,2}$ MARCUS MISSAL, ${ }^{2}$ AND PHILIPPE LEFÈVRE ${ }^{1,2}$ \\ ${ }^{1}$ Centre for Systems Engineering and Applied Mechanics, Université Catholique de Louvain, 1348 Louvain-la-Neuve; \\ and ${ }^{2}$ Laboratory of Neurophysiology, Université Catholique de Louvain, 1200 Brussels, Belgium
}

Submitted 14 August 2002; accepted in final form 15 November 2002

\begin{abstract}
Blohm, Gunnar, Marcus Missal, and Philippe Lefèvre. Interaction between smooth anticipation and saccades during ocular orientation in darkness. J Neurophysiol 89: 1423-1433, 2003. First published November 20,2002; 10.1152/jn.00675.2002. A saccade triggered during sustained smooth pursuit is programmed using retinal information about the relative position and velocity of the target with respect to the eye. Thus the smooth pursuit and saccadic systems are coordinated by using common retinal inputs. Yet, in the absence of retinal information about the relative motion of the eye with respect to the target, the question arises whether the smooth and saccadic systems are still able to be coordinated possibly by using extraretinal information to account for the saccadic and smooth eye movements. To address this question, we flashed a target during smooth anticipatory eye movements in darkness, and the subjects were asked to orient their visual axis to the remembered location of the flash. We observed multiple orientation saccades (typically 2-3) toward the memorized location of the flash. The first orienting saccade was programmed using only the position error at the moment of the flash, and the smooth eye movement was ignored. However, subsequent saccades executed in darkness compensated gradually for the smooth eye displacement (mean compensation $\cong 70 \%$ ). This behavior revealed a $400-\mathrm{ms}$ delay in the time course of orientation for the compensation of the ongoing smooth eye displacement. We conclude that extraretinal information about the smooth motor command is available to the saccadic system in the absence of visual input. There is a 400-ms delay for smooth movement integration, saccade programming and execution.
\end{abstract}

\section{N T R O D U C T I O N}

Saccades and smooth pursuit eye movements are used in combination during orientation of the visual axis toward a moving target. It was previously thought that these different eye movements were controlled by independent neural systems, although they act in synergy to reach a moving target. This view has recently been challenged at the neuronal level (Krauzlis and Miles 1998; Missal and Heinen 2001; Missal and Keller 2002; Missal et al. 2000). At the behavioral level, the coordination between saccades and smooth pursuit has so far been studied during orientation toward a moving visual target (de Brouwer et al. 2001, 2002). In this condition, it has been shown that saccadic and smooth pursuit motor commands sum up. Furthermore, the saccadic and smooth pursuit systems could share a common source of information, i.e., the slip of the target image on the retina. This sharing of visual informa-

Address for reprint requests: P. Lefèvre, CESAME, Université Catholique de Louvain, 4, avenue G. Lemaître, 1348 Louvain-la-Neuve, Belgium (E-mail: lefevre@csam.ucl.ac.be). tion allows the saccadic system to compensate for the motion of the target during the latency period and the execution of catch-up saccades. Thus to accurately orient the eyes toward a visual target, the saccadic and smooth pursuit systems interact, and movements are programmed using retinal information. Yet, this is only true if there is continuous visual feedback. In the absence of visual input, the question arises whether different movements could still be coordinated. In such a situation, the oculomotor system has to integrate extraretinal signals to account for self-motion.

The role of extraretinal signals in the saccadic system has been addressed in numerous studies by means of the doublestep and the colliding saccades paradigms (Aslin and Shea 1987; Becker and Jürgens 1979; Dassonville et al. 1992; Dominey et al. 1997; Goossens and Van Opstal 1997; Hallett and Lightstone 1976a,b; Mays and Sparks 1980; Mushiake et al. 1999; Schlag and Schlag-Rey 1990; Schlag et al. 1989, 1990; Schlag-Rey et al. 1989; Tian et al. 2000). In these studies, saccades toward the memorized position of flashed targets were investigated. If before a saccade the eyes were deviated by another saccade evoked either visually or by microstimulation, the second saccade accurately reached its goal. The system had to use some information about the first eye movement to adjust the second saccade because the initial retinotopic vector of the second saccade was not accurate anymore. These authors concluded that the saccadic system has access to extraretinal signals, i.e., the efference copy of the saccadic motor command, that update the internal representation of the target in space. Thus in the absence of retinal input, the saccadic system makes an extensive use of extraretinal signals.

However, the double-step and colliding saccades paradigms investigate only one aspect of the self-movement integration, i.e., whether the oculomotor system keeps track of consecutive saccades by using extraretinal signals. What would happen if prior to a saccade the eyes were displaced by a smooth eye movement instead of a saccade? Would the system have access to extraretinal information about self-movement to accurately orient the eyes? If this was the case, could the system adjust the saccadic goal or would there be an a posteriori mechanism that accounted for the smooth perturbation? Both scenarios would need a source of extraretinal information to compensate for the

\footnotetext{
The costs of publication of this article were defrayed in part by the payment of page charges. The article must therefore be hereby marked "advertisement" in accordance with 18 U.S.C. Section 1734 solely to indicate this fact.
} 
smooth eye displacement. Here, we address the question whether the saccadic system receives such extraretinal information from the pursuit system to account for smooth eye movements in darkness.

To investigate this topic, we used a paradigm that could generate smooth eye movements without bringing into play any retinal slip information. This allowed us to rule out the hypothesis that a memorized retinal slip signal could play a role. Furthermore, our protocol provided a saccadic goal using as little retinal information as possible. We achieved this objective by using anticipatory smooth pursuit and memoryguided saccades. Indeed, orientation of the visual axis in the absence of retinal stimulation is possible in both smooth and saccadic systems. Saccades can be aimed toward the memorized position of a previously flashed target (Goldman-Rakic 1987). Anticipatory smooth eye movements can be evoked in the absence of a moving target if there is a previous "build-up" of the expectation of future target motion (Barnes and Asselman 1991; Kao and Morrow 1994; Kowler et al. 1984). Thus we reduced the visual information available to the oculomotor system to a minimum. This disabled the ability of the saccadic system to rely on retinal information about motion to program saccades. In that way, we created an original paradigm that allowed us to investigate the hypothesis of a mechanism that could compensate for the smooth eye movements by means of corrective saccades based on extraretinal signals.

\section{METHODS}

\section{Experimental set-up}

Human subjects sat in darkness in front of a 1-m distant tangent screen, which spanned about $\pm 45^{\circ}$ of their visual field. Their head was restrained by a chin-rest. A $0.2^{\circ}$ red LASER target spot was back-projected onto the screen and moved horizontally under the control of a mirror-galvanometer. Movements of one eye were recorded with the scleral coil technique, Skalar Medical BV (Collewijn et al. 1975; Robinson 1963). Healthy subjects without any known oculomotor abnormalities were recruited after informed consent. Among the seven subjects, three were completely naïve of oculomotor experiments. Mean age was 29, ranging from 22 to 36 . All procedures were conducted with approval of the Universite Catholique de Louvain Ethics committee.

\section{Paradigm}

Recording sessions were composed of a series of blocks of 40 trials. Each session was divided into three parts: first, each subject had to perform one block of control trials toward stationary targets, then a block of build-up trials was presented to build up a smooth anticipatory response, and the last (but longest) part of the sessions was composed of several blocks of test trials mixed with build-up trials.

Control trials were composed of two types of randomly presented stimuli (transient and sustained; Fig. 1A). All control trials began with a fixation period of $800 \mathrm{~ms}$ in the center of the screen. After the target disappeared for a variable duration of 100-500 ms (gap), either a 10 -ms flash (transient control condition) or a 1,000-ms target (sustained control condition) was presented at random locations in a range $\pm 15^{\circ}$ around the central fixation point. All control trials lasted for 2,300 ms. Subjects were instructed to orient their eyes toward the target (sustained control condition) or toward the remembered position of the flash (transient control condition).

To build up a smooth anticipatory response, we used build-up trials (Fig. $1 B$ ). After a fixation period of $800 \mathrm{~ms}$ in the center of the screen, the target disappeared for $300 \mathrm{~ms}$. The gap duration was chosen to
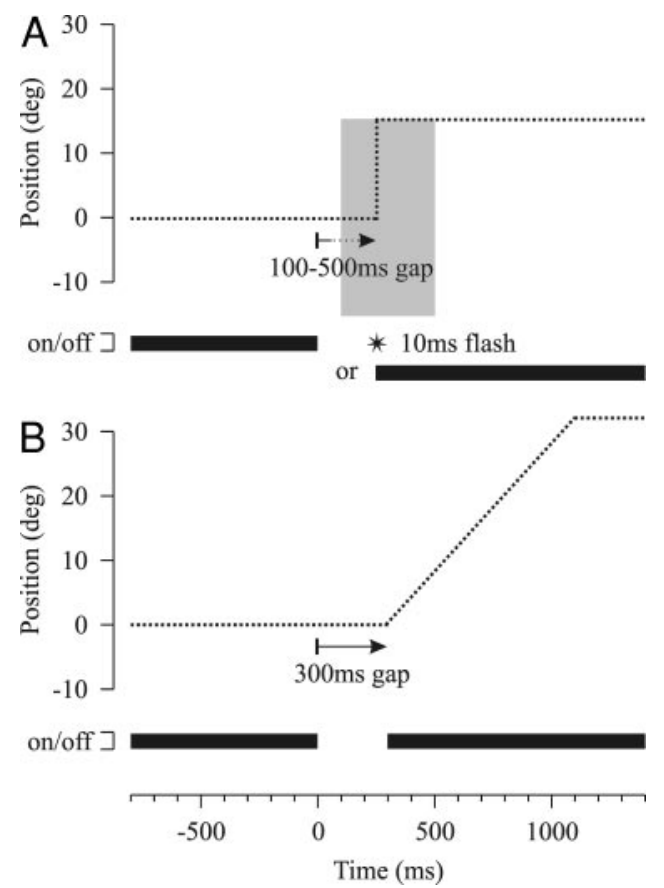

FIG. 1. Experimental paradigm. A: test trial condition. The trial starts with an 800-ms fixation period. Afterward, either a 10-ms flash (transient condition) or a 1,000-ms fixation (sustained condition) is presented at random time and position. $\square$, indicated the randomization zone. The gap varies continuously between 100 and $500 \mathrm{~ms}$, and the target reappears randomly in a range $\pm 15^{\circ}$ around the expected target position. $\cdots$, target position; $\mathbf{\square}$, the presence of the target; *, for the 10-ms flash. B: build-up trial condition. After 800-ms fixation, the target disappears for $300 \mathrm{~ms}$ (gap) and reappears moving for another 800 $\mathrm{ms}$ at $40 \%$ s to the right followed by $500 \mathrm{~ms}$ of final fixation.

give a maximal smooth anticipatory response (Morrow and Lamb 1996). At reappearance, the target moved for $800 \mathrm{~ms}$ from the center of the screen at $40 \% \mathrm{~s}$ always in the same direction. The trial ended with a 500-ms fixation period. Subjects were instructed to follow the target as accurately as possible.

For the third part of the recording session, build-up trials were randomly interleaved with $30 \%$ of test trials: transient and sustained test trials (Fig. 1A). Both test conditions began like the build-up trials with an 800-ms fixation at the center of the screen followed by a gap that varied randomly in duration from 100 to $500 \mathrm{~ms}$. After the gap, either a 10-ms flash (transient test condition) or a 1,000-ms target (sustained test condition) was presented at a random position in a range $\pm 15^{\circ}$ around the expected target position ( = target position of build-up trial). All trials lasted for 2,300 ms. Subjects were instructed to follow the target as accurately as possible and to fixate the memorized target position in case of a transient test trial.

\section{Data acquisition and analysis}

Eye and target position were sampled at $500 \mathrm{~Hz}$ and stored on the hard disc of a PC for off-line analysis. MATLAB (Mathworks) was used to implement digital filtering, velocity and acceleration estimation algorithms. Position signals were low-pass filtered by a zerophase digital filter (autoregressive forward-backward filter, cutoff frequency: $50 \mathrm{~Hz}$ ). Velocity and acceleration were derived from position signals using a central difference algorithm.

In our analysis, only control and test trials were analyzed. We were interested in saccades directed toward the flashed or sustained target. Saccades were detected using an acceleration threshold of $750 \% \mathrm{~s}^{2}$, and their latency was measured with respect to the target onset. We analyzed the first saccade for all stimulus conditions. Up to five orienting saccades were taken into account in the transient condition. 
Saccades were removed from the eye velocity trace to obtain the smooth velocity. Therefore we measured the smooth eye velocity before and after the saccade and interpolated linearly between the values to obtain an estimation of the smooth eye velocity during the saccades. This allowed us to quantify the contribution of the smooth pursuit system $P_{\text {Amp }}$ to the total saccadic amplitude $S_{\text {Amp }}$. We also measured different parameters that may play a role in saccadic programming. Position error (PE) and retinal slip (RS) signals were sampled at the moment of the target onset (to) and $100 \mathrm{~ms}$ before the saccade. For more details about the estimation of those parameters, see METHODS section of de Brouwer et al. (2002). Furthermore, the smooth eye velocity signal was integrated to obtain the smooth eye displacement SED. In the transient test condition, the orientation process continued after the first saccade, and the time course of this process was investigated. The final orientation was defined as the eye position after the last saccade before return to the central fixation point.

\section{RES U L TS}

Three examples of the different stimulus conditions are illustrated in Fig. 2. For build-up trials (Fig. 2A), the eye movement could be entirely smooth although most of the time anticipatory and/or visually guided saccades were present. In the test trials (Fig. 2, $B$ and $C$ ), subjects anticipated as in the build-up trials. Sustained test trails (Fig. 2B) typically presented one or two saccades toward the target, whereas for transient test trials (Fig. 2C) subjects typically needed two or three orienting saccades. Subjects reported that they perceived the 10-ms flash as being stationary. This is in accordance with the findings of Gellman and Fletcher (1992). However, sustained test targets were perceived to be in movement, which is due to the retinal slip caused by the smooth anticipatory eye movement. The last saccade toward the visual or remembered target always occurred when smooth eye velocity was close to $0 \%$ s.

Figure 3 shows the pattern of smooth eye movements for transient test trials. The pattern was very similar for sustained

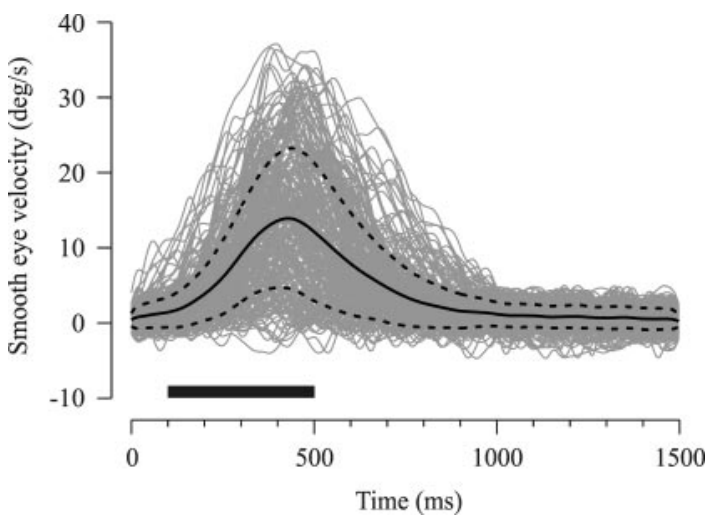

FIG. 3. Time course of smooth eye movements for the transient test condition. Gray lines show individual trials. The mean smooth eye movement (solid line) and the associated SD (dashed lines) are also shown. The bar at the bottom of the figure indicates the range of the time of appearance of the flash. All smooth eye movement traces are aligned on the gap onset (time 0 ).

test trials. After the gap onset (Fig. 3, time 0), smooth anticipation built up. The mean smooth anticipatory eye velocity at the moment of the target reappearance (sustained or transient) was $9.3 \pm 6.3 \%(n=4,238)$ ranging from 0 to $34 \%$. The amplitude of the smooth anticipatory movement varied from trial to trial and depended on the trials history across the experimental session (build-up or test trials). This influence of the history of previous trials has first been described by Kowler et al. (1984).

\section{General saccadic properties}

In this section, we describe the characteristics of the first saccade toward the test target for both the sustained and the transient test conditions. All results were tested and are valid separately for each condition but will be presented together for the sake of clarity. We first analyzed the main sequence relationship as well as the saccadic latency histogram.
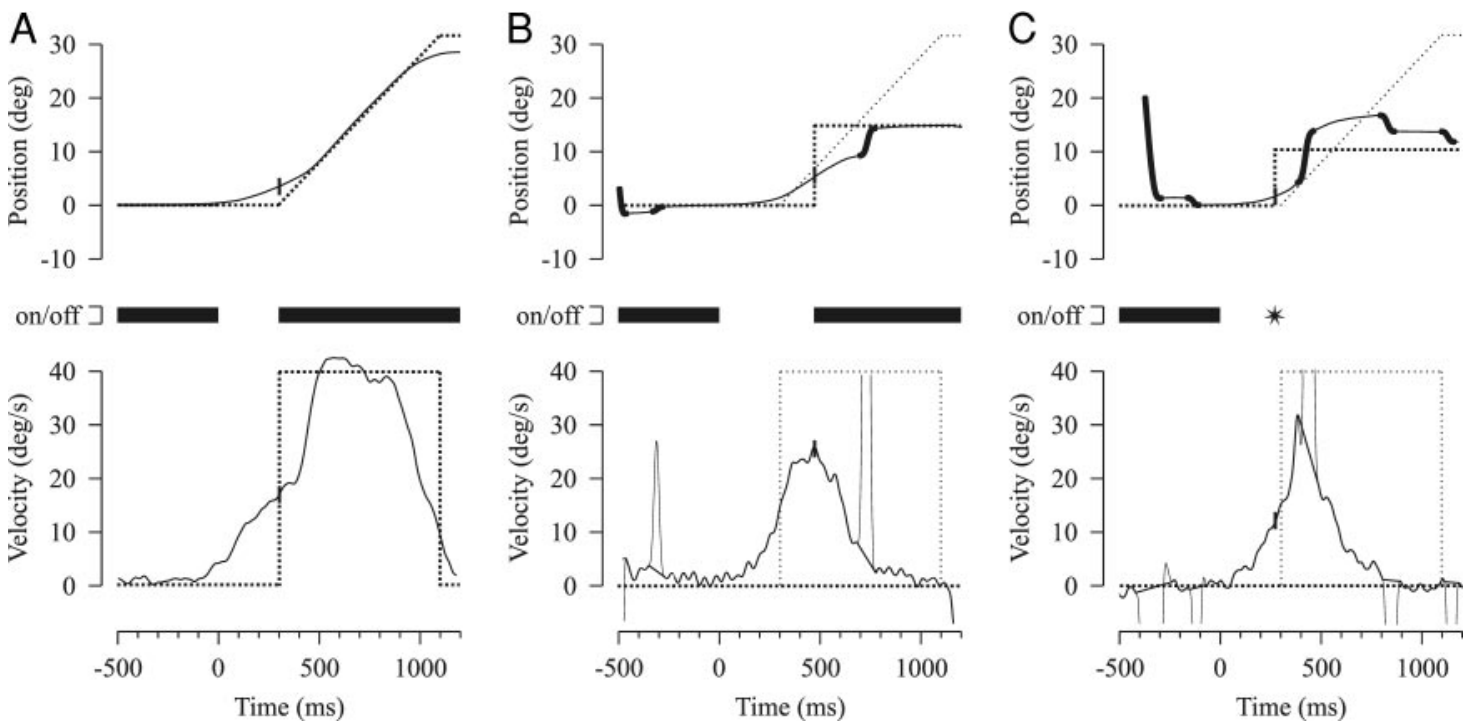

FIG. 2. Typical examples. Top: solid lines represent the eye position (bold lines mark saccades), dotted lines are target position, and thin dotted lines stand for the expected build-up target position (in $B$ and $C$ ). Bottom: solid lines represent the smooth eye velocity (without saccades). Saccades are shown as thin solid lines. Dotted lines represent target velocity and thin dotted lines stand for the expected build-up target velocity (in $B$ and $C$ ). The horizontal bars in the center part of $A-C$ represent the presence of the target; the star in the center part of $C$ stands for the 10-ms flash. $A$ : build-up response. $B$ : sustained test trial. $C$ : transient test trial. 

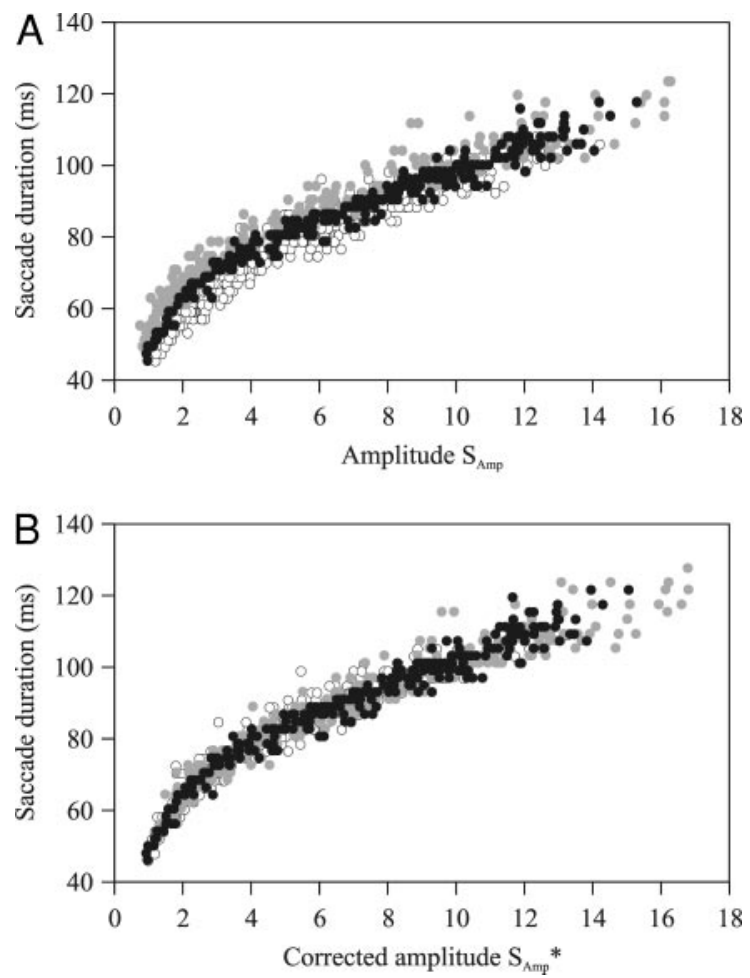

FIG. 4. Main sequence relationship between saccade duration and amplitude. Forward saccades (saccades and smooth movements in the same direction,, ), reverse saccades (saccades and smooth movements in opposite directions, $\bigcirc$ ), and control saccades $(\bullet)$ are represented. $A$ : the main sequence between duration and amplitude before correction $\left(S_{\mathrm{Amp}}\right)$. Forward, reverse, and control saccades fall in 3 distinct populations. $B$ : the same main sequence for the corrected amplitude $S_{\text {Amp }}^{*}$, i.e., after correction for the smooth movement $\left(P_{\text {Amp }}\right)$. The 3 populations merge into 1 .

During sustained smooth pursuit, the motor commands of the saccadic and smooth pursuit system sum up (de Brouwer et al. 2002). As the smooth pursuit system is active during the saccadic command execution, the total saccadic amplitude
$S_{\mathrm{Amp}}=S_{\mathrm{Amp}}^{*}+P_{\mathrm{Amp}}$, where $S_{\mathrm{Amp}}^{*}$ is the component from the saccadic system and $P_{\text {Amp }}$ is the contribution of the pursuit system. This is reflected in the saccadic main sequence relationship, where control saccades and those during sustained pursuit (opposite or in the same direction as the saccadic command) fall into three different populations. After correction for the participation of the smooth pursuit system, all three populations merge into one. Here, we performed this analysis for saccades triggered during smooth anticipatory eye movements. Both main sequence relationships-saccade duration versus saccade amplitude and saccade peak velocity versus saccade amplitude — were analyzed. Correlations for both main sequence relationships were significantly better ( $t$-test, $P<$ $0.05)$ after correction for the smooth anticipation component than before correction (total $n=4,985$ ). Figure 4 illustrates this result for the main sequence relationship between saccade duration and amplitude for subject 7. Only for one subject (subject 5) did the second main sequence relationship not show a significant improvement after correction for the smooth component. Taking it all together, we showed here that like smooth pursuit, smooth anticipation adds up to the saccadic motor command. Therefore when analyzing saccade programming, we first removed the pursuit component $\left(P_{\text {Amp }}\right)$ from the saccade $\left(S_{\text {Amp }}\right)$. All subsequent analyses were thus performed on the corrected saccade amplitude $S_{\text {Amp }}^{*}$.

For the first orientation saccade after target reappearance, we evaluated whether the information used for its programming was based on the sensory signal of the target or whether it was an anticipatory saccade directed toward the expected moving target. Figure $5 A$ shows an example of such an anticipatory saccade (latency: $37 \mathrm{~ms}$ ). This analysis was done to quantify the minimum saccade latency we could consider for the analysis of saccades during test trials. If the saccade endpoint fell into a $\pm 5^{\circ}$ interval around the target position, the saccade was considered to be visually driven. Otherwise, if the saccade endpoint fell into another $\pm 5^{\circ}$ interval around the expected
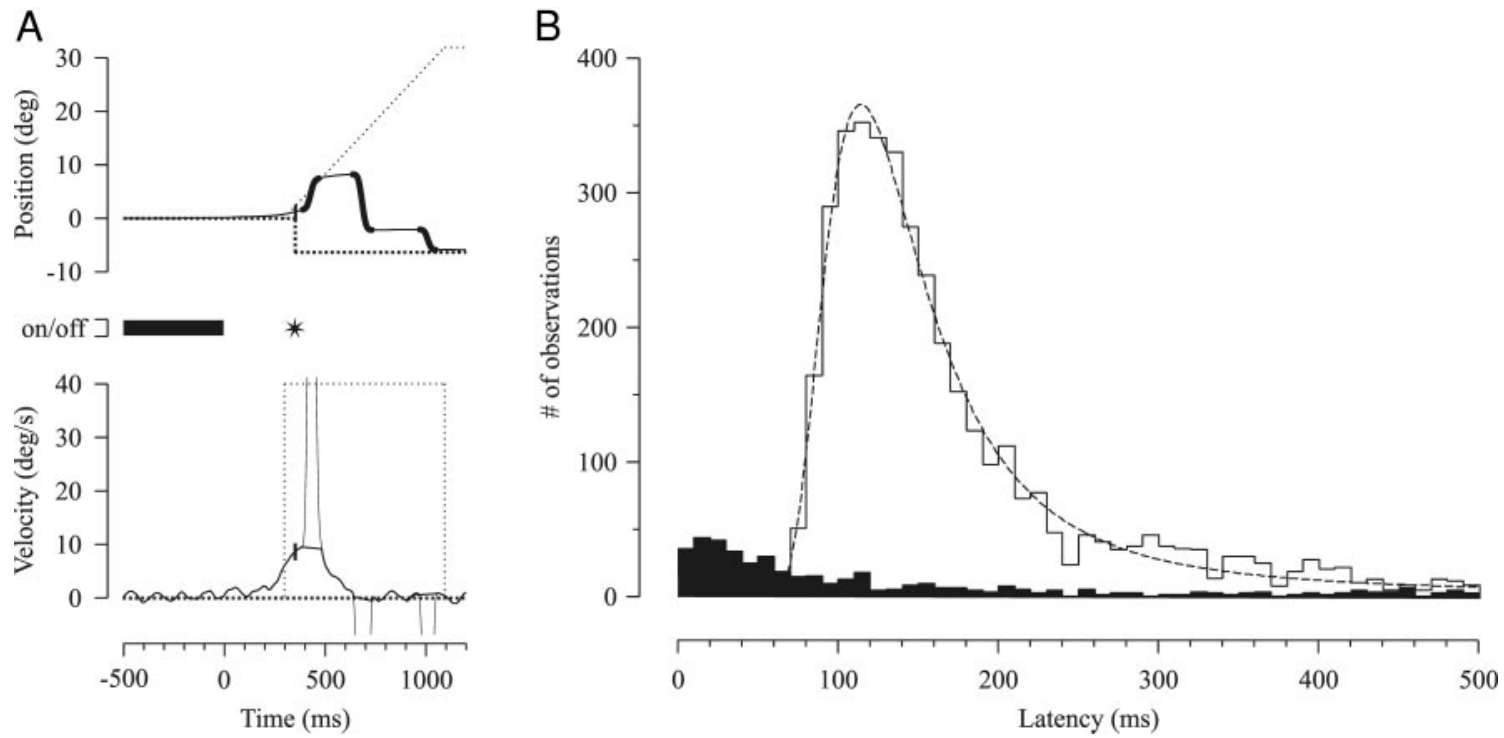

FIG. 5. Minimum latency of the 1st saccade. $A$ : anticipatory saccade directed toward the expected target ramp (latency: 37 ms). The same conventions as in Fig. 2 are used. $B$ : histogram (10-ms bins) of the trials for which the saccade was programmed toward the actual target $(\square, n=3,832)$ or to the expected target $(\mathbf{\square}, n=406)$. Data are pooled for all subjects. - - - the recinormal distribution fit of the LATER model for saccade latency (Reddi and Carpenter 2000). Fitted parameters of the normal distribution in the reciprocal time domain are $8.77 \pm 2.38 \mathrm{~s}^{-1}$. 
build-up ramp, the saccade was classified as anticipatory. If the saccade endpoint fell into both intervals, the trial was not classified (6.5\% of trials). The histogram of the classified saccadic latencies pooled for all subjects is shown in Fig. 5B; - represents anticipatory saccades $(n=406)$, whereas $\square$ corresponds to visually guided saccades $(n=3,832)$. Saccades that fell into - for latencies $>300$ ms came exclusively from the transient test condition. To describe the latency histogram with an analytical function, we first tried a normal distribution. However, the Jarque-Bera test for goodness of fit to a normal distribution rejects the hypothesis that the saccadic latencies follow a normal distribution $(P<0.001)$. Thus we described the latency histogram with a recinormal function (Reddi and Carpenter 2000). This function fitted well the data (see - - - in Fig. $5 B ; R=0.972, P<0.001$ ), which means that it is the reciprocal of latency that follows a normal distribution. The maximum of the fitted recinormal distribution lies at $114 \mathrm{~ms}$. The onset of visually guided saccades in the histogram reveals saccadic latencies as short as $80 \mathrm{~ms}$. No significant difference was found between the sustained and the transient test conditions. The maximum of the fitted distribution varied from 88 to $132 \mathrm{~ms}$ across subjects.

\section{Programming of the first saccade}

We were interested in how the saccadic system programs the first orienting saccade after the target reappearance for both the sustained and transient test conditions. In the previous section, we showed that the smooth anticipatory command adds linearly to the motor command of the saccadic system. Thus to analyze the saccade programming, we subtracted the smooth anticipatory eye displacement during the saccade $P_{\text {Amp }}$ from the measured saccade amplitude $S_{\mathrm{Amp}}$ to obtain the purely saccadic component $S_{\mathrm{Amp}}^{*}$. In this analysis, we included saccades with latency $>125 \mathrm{~ms}$ (de Brouwer et al. 2002). Table 1 summarizes the principal parameters that characterize the sustained and transient test trials. The saccadic gain was defined as the ratio between the measured saccade amplitude $S_{\mathrm{Amp}}$ and the ideal saccade $\left(S_{\mathrm{Amp}}+\mathrm{PE}_{\text {after 1st saccade }}\right)$. For the transient test trials, Table 1 gives also an indication about the final error

TABLE 1. Mean values and ranges of different parameters that characterize our data set

\begin{tabular}{|c|c|c|c|}
\hline Variable & Values & $\begin{array}{c}{[25-75] \%} \\
\text { Range }\end{array}$ & $n$ \\
\hline \multicolumn{4}{|l|}{ Sustained } \\
\hline$\left|S_{\text {Amp }}^{*}\right|$, deg & $5.633 \pm 3.919$ & [2.298-8.544] & 984 \\
\hline$\left|\mathrm{PE}_{-100}\right|$, deg & $5.870 \pm 4.326$ & [2.107-9.292] & 984 \\
\hline $\mathrm{RS}_{-100}, \mathrm{deg} / \mathrm{s}$ & $10.225 \pm 8.538$ & [3.771-14.916] & 984 \\
\hline$\left|\mathrm{PE}_{\text {after 1st saccade }}\right|$, deg & $0.806 \pm 0.697$ & {$[0.289-1.102]$} & 984 \\
\hline Saccadic gain & $0.929 \pm 0.182$ & [0.834-1.043] & 984 \\
\hline \multicolumn{4}{|l|}{ Transient } \\
\hline$\left|S_{\text {Amp }}^{*}\right|, \operatorname{deg}$ & $6.330 \pm 3.478$ & [3.287-8.917] & 583 \\
\hline$\left|\mathrm{PE}_{\mathrm{to}}\right|$, deg & $7.008 \pm 3.867$ & [3.515-10.073] & 583 \\
\hline $\mathrm{EV}_{\text {to }}, \mathrm{deg} / \mathrm{s}$ & $9.387 \pm 6.188$ & [4.820-14.716] & 583 \\
\hline $\mathrm{SED}_{\text {after 1st saccade, }}$ deg & $2.229 \pm 1.682$ & {$[0.876-3.384]$} & 583 \\
\hline$\left|\mathrm{PE}_{\text {after 1st saccade }}\right|$, deg & $2.019 \pm 1.437$ & {$[0.851-2.918]$} & 583 \\
\hline Saccadic gain & $0.880 \pm 0.328$ & [0.684-1.018] & 583 \\
\hline$\left|\mathrm{PE}_{\text {end }}\right|$, deg & $1.069 \pm 1.434$ & [0.114-1.556] & 1354 \\
\hline $\mathrm{SED}_{\text {total }}$, deg & $2.956 \pm 3.393$ & {$[1.120-4.540]$} & 1354 \\
\hline
\end{tabular}

Values are means $\pm \mathrm{SD}$.
TABLE 2. Correlation coefficients for the multiple regression analysis between the dependent variable $S_{A m p}^{*}$ and the independent variables for sustained test trials

\begin{tabular}{|c|c|c|c|c|}
\hline \multirow{2}{*}{$\begin{array}{c}\text { Independent } \\
\text { Variable } 1\end{array}$} & \multirow{2}{*}{$\begin{array}{c}\text { Independent } \\
\text { Variable } 2\end{array}$} & \multirow[b]{2}{*}{$R$} & \multicolumn{2}{|c|}{ Partial $R$} \\
\hline & & & Variable 1 & Variable 2 \\
\hline $\mathrm{PE}_{-100}$ & 一 & $0.990(<0.01)$ & - & - \\
\hline $\mathrm{RS}_{-100}$ & - & $0.185(<0.01)$ & - & - \\
\hline $\mathrm{PE}_{-100}$ & $\mathrm{RS}_{-100}$ & $0.991(<0.01)$ & $0.990(<0.01)$ & $0.340(<0.01)$ \\
\hline
\end{tabular}

$P$ values are in parentheses. $n=984$.

$\mathrm{PE}_{\text {end }}$ and the total smooth eye displacement $\mathrm{SED}_{\text {total }}$ at the end of the orientation process.

In the case of the sustained test condition, the question is whether the retinal slip is evaluated to program the saccade, as is the case during sustained pursuit (de Brouwer et al. 2002). Indeed, de Brouwer et al. (2002) showed that saccades triggered during sustained pursuit are programmed using an estimate of the position error and the retinal slip measured $100 \mathrm{~ms}$ before saccade onset. They hypothesized that $100 \mathrm{~ms}$ before saccade onset is the last moment for visual information to be taken into account in saccadic amplitude programming (Becker and Jürgens 1979; Heywood and Churcher 1981). To test the hypothesis that the system behaves in the same way for anticipatory and visually guided smooth pursuit, we performed a multiple regression analysis for the dependent variable $S_{\mathrm{Amp}}^{*}$ using the independent variables $\mathrm{PE}_{-100}$ and $\mathrm{RS}_{-100}$. The index -100 indicates that we measured these parameters $100 \mathrm{~ms}$ before saccade onset. Table 2 shows the results of the analysis for the sustained test condition. The best correlation was obtained with $\mathrm{PE}_{-100}$ and $\mathrm{RS}_{-100}$ as independent variables (Eq. 1)

$S_{\mathrm{Amp}}^{*}=0.249+0.930 \cdot \mathrm{PE}_{-100}+0.059 \cdot \mathrm{RS}_{-100} \quad(R=0.991, n=984)$

The separate analysis for each subject shows that the multiple regression with $\mathrm{PE}_{-100}$ and $\mathrm{RS}_{-100}$ was always significant, except for one subject (subject 5: $P>0.05$ for coefficient of $\mathrm{RS}_{-100}$ ). Across subjects, regression coefficients varied for $\mathrm{PE}_{-100}$ between 0.885 and 0.984 and for $\mathrm{RS}_{-100}$ between 0.035 and 0.086 . As a result, we showed here that the same strategy is used for saccades to sustained targets during smooth pursuit or during smooth anticipation.

In the transient test condition, the target was only presented very briefly (for $10 \mathrm{~ms}$ ), and therefore the system did not have time to evaluate the retinal slip. Furthermore, after the flash, there was no more visual feedback that could be used to program the orienting saccades. The only available sensory information was the memorized position error of the target at the moment of the flash onset $\mathrm{PE}_{\mathrm{to}}$. Thus the question here is whether the saccadic system has access to any other internal information such as, for example the smooth eye velocity at the moment of the flash onset $\mathrm{EV}_{\text {to }}$ or the smooth eye displacement SED (= integral of smooth eye velocity) between the flash and the saccade onset. To test these hypotheses, we performed a multiple regression analysis with the dependent variable $S_{\mathrm{Amp}}^{*}$ and the independent variables $\mathrm{PE}_{\mathrm{to}}, \mathrm{EV}_{\mathrm{to}}$, and SED. Only saccades with latencies $<250 \mathrm{~ms}$ were considered in this analysis. Table 3 summarizes the results of this analysis. Single regression results showed that the saccade amplitude was best 
TABLE 3. Correlation coefficients for the multiple regression analysis between the dependent variable $S_{A m p}^{*}$ and the independent variables for transient test trials

\begin{tabular}{ccccc}
\hline \hline & & & \multicolumn{2}{c}{ Partial $R$} \\
\cline { 4 - 5 } Independent \\
Variable 1 & $\begin{array}{c}\text { Independent } \\
\text { Variable 2 }\end{array}$ & $R$ & Variable 1 & Variable 2 \\
\hline PE $_{\text {to }}$ & - & $0.989(<0.01)$ & - & - \\
$\mathrm{EV}_{\text {to }}$ & - & $0.255(<0.01)$ & - & - \\
$\mathrm{SED}$ & - & $0.254(<0.01)$ & - & - \\
$\mathrm{PE}_{\text {to }}$ & $\mathrm{EV}$ & $0.990(<0.01)$ & $0.989(<0.01)$ & $0.034(>0.05)$ \\
$\mathrm{PE}_{\text {to }}$ & $\mathrm{SED}$ & $0.990(<0.01)$ & $0.989(<0.01)$ & $0.074(>0.05)$ \\
\hline
\end{tabular}

$P$ values are in parentheses. $n=583$.

correlated with $\mathrm{PE}_{\mathrm{to}}$. Partial correlation coefficients in the multiple regression analysis were significant for $\mathrm{PE}_{\mathrm{to}}$ but neither for $\mathrm{EV}_{\text {to }}$ nor for SED. Clearly, our statistical analysis showed that there was no other parameter than $\mathrm{PE}_{\mathrm{to}}$ that was used for the first saccade programming in the transient test condition (Eq. 2)

$$
S_{\mathrm{Amp}}^{*}=-0.183+0.888 \cdot \mathrm{PE}_{\mathrm{to}} \quad(R=0.989, n=583)
$$

Regression coefficients for $\mathrm{PE}_{\text {to }}$ varied for each subject and ranged from 0.807 to 0.947 . In the transient test condition, there was no significant difference in gain of the first saccade between the transient test condition and the transient control trials $(P>0.05)$. This indicates that for flashed targets, the smooth eye movement was ignored by the saccadic system. This was compatible with the error measured after the saccade, which was proportional to the smooth eye displacement (SED) between the flash and the end of the saccade (Fig. 6A). When plotting data for all subjects, the equation of the first-order regression was the following

$$
\mathrm{PE}=0.014+1.139 \cdot \mathrm{SED} \quad(R=0.665, n=583)
$$

The slope of the regression varied between subjects from 0.772 to 1.260 ( $n=62$ to 123 ). Figure $6 B$ provides a comparison with control trials $(-0.080 \pm 1.444 \mathrm{deg}, n=1,251$; mean $\pm \mathrm{SD})$. In the transient test condition, the dependence of the error after the first saccade on SED confirmed that at this time SED was not used by the saccadic system to program the saccade.

\section{Time course of orientation}

Until now, we only analyzed the first orienting saccade that occurred after the target reappearance. In the case of the sustained test condition, most of the time the first orienting saccade brought the eye exactly onto the target. If there was a residual error, it was corrected by a second saccade, and the orientation process was completed. Thus no further analysis of the sustained test situation was necessary. But in the case of the transient test condition, our typical example (Fig. 2C) clearly shows that the orientation process went on after the first saccade and that subsequent saccades contributed significantly to the final gaze orientation. In this case, how did the oculomotor system perform this orientation process without any additional visual feedback?

We first quantified the accuracy of the final orientation. For the orientation to be accurate, the subject had to compensate for the total smooth eye displacement after the flash. Figure $7 \mathrm{~A}$ shows the error after the last orienting saccade $\mathrm{PE}_{\text {end }}$ as a function of the total smooth eye displacement between the flash and the moment of the final orienting saccade $\mathrm{SED}_{\text {total }}$. The first order regression for all subjects pooled together (dashed) follows the equation

$$
\mathrm{PE}_{\text {end }}=0.027+0.308 \cdot \mathrm{SED}_{\text {total }} \quad(R=0.328, n=1354)
$$

Across subjects, the slope ranged from 0.075 to $0.572(n=$ 168-370). In Fig. 7A, $\cdots$ corresponds to the regression in Fig. $6 A$ and allows a direct comparison between compensation for SED after the first saccade versus at the end of the orientation process. This confirmed that the orientation process did not stop after the first saccade and that the final orientation accounted for most of the smooth eye displacement. We can get an idea of the time course of orientation by providing additional regression lines for two intermediate steps between the first saccade and the final orientation. This is shown in Fig. $7 B$. -.. and - - correspond to regression lines from Fig. 7A. The slopes of the regression lines at 500 and $750 \mathrm{~ms}$ after the flash were $0.793(R=0.598, n=1,272)$ and $0.418(R=0.469, n=$ $1,345)$, respectively. This indicated that the smooth eye displacement was not accounted for in one step but that there was a gradual orientation process. A more detailed representation of this gradual orientation is provided in Fig. 7C. The evolution of the time course of orientation error is presented as a function of the smooth eye displacement SED between 200 and 1,000 $\mathrm{ms}$ in 50-ms steps.

In the following section, the time course of the orientation process will be analyzed in more detail. Therefore position error $\operatorname{PE}(t)$ and smooth eye displacement $\operatorname{SED}(t)$ were sampled at regular 50-ms intervals, and a second-order regression analysis was performed using the sampled position error as dependent variable and the sampled smooth eye displacement SED and the position error at the moment of the flash $\mathrm{PE}_{\text {to }}$ as independent variables

$$
\operatorname{PE}(t)=\beta(t)+\gamma(t) \cdot \mathrm{PE}_{\mathrm{to}}+\delta(t) \cdot \operatorname{SED}(t)
$$

$\mathrm{PE}_{\text {to }}$ was included in the regression to investigate whether the error due to a saccadic gain $<1$ (after the first saccade) was compensated later in the orientation process. The regression coefficients $\gamma(t)$ and $\delta(t)$ for $\mathrm{PE}_{\text {to }}$ and SED respectively are shown in Fig. $8 A$. From the SED coefficient $\delta(t)$, one can see that the orientation process started $\sim 400 \mathrm{~ms}$ after the flash and
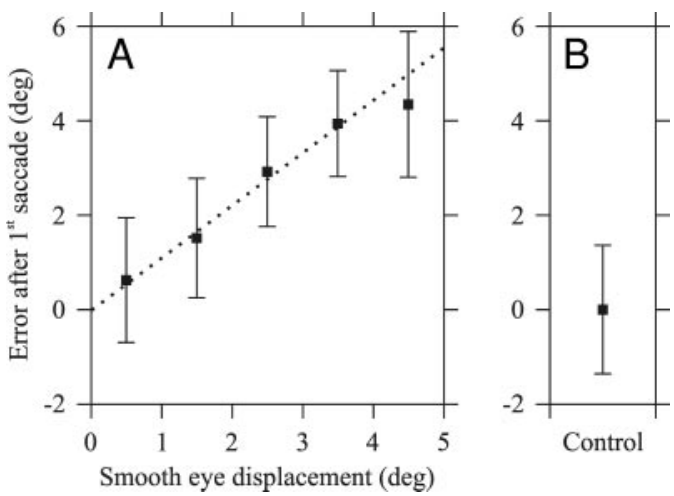

FIG. 6. Position error after the 1 st saccade (latency $<250 \mathrm{~ms}$ ) as a function of the smooth eye displacement SED (mean $\pm \mathrm{SD}$ ). A: transient test trials. $\cdots$, fitted on raw data. The number of points in each $1^{\circ}$ bin varies between 47 and 233. $B$ : control trials for comparison (SED $=0^{\circ}, n=1,251$ ). 

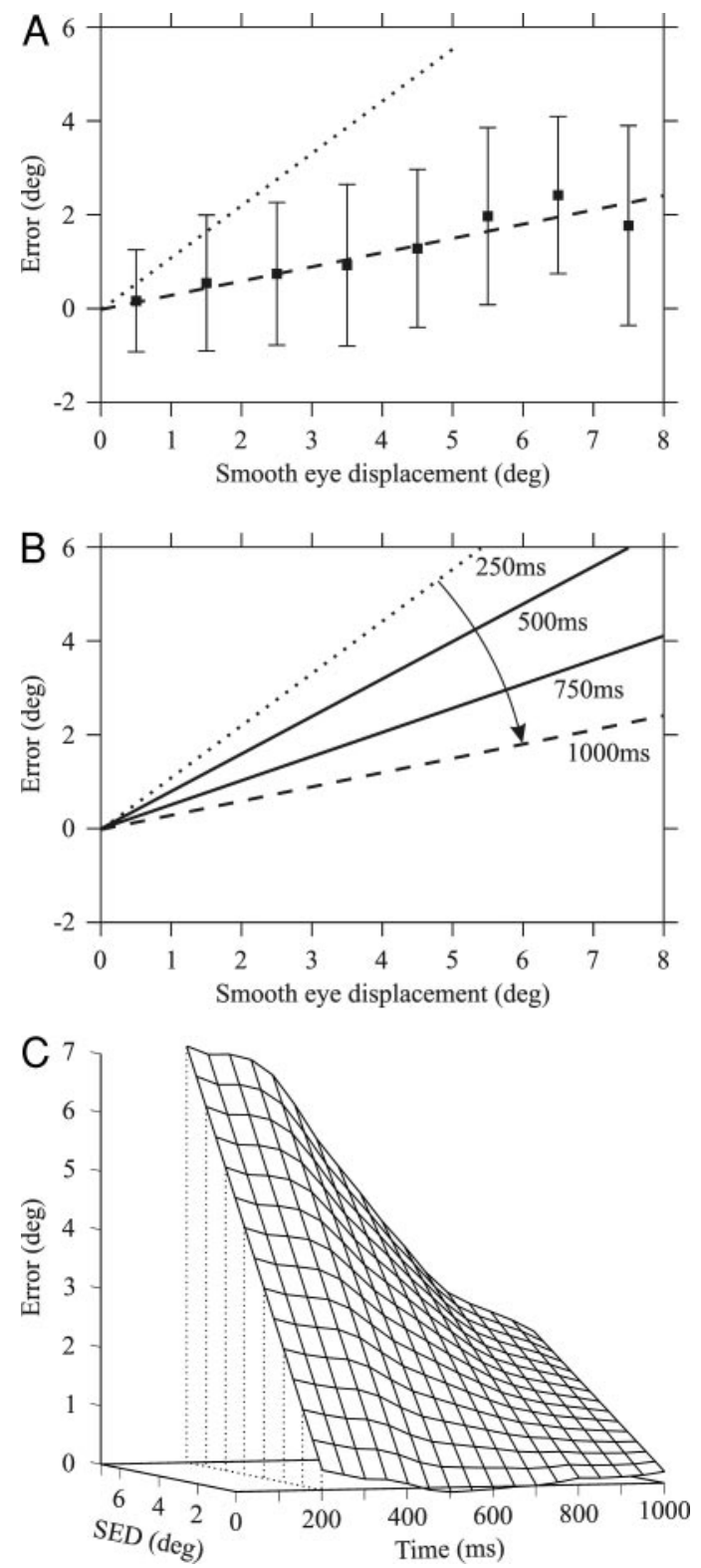

FIG. 7. Time course of position error. A: final error as a function of the total smooth eye displacement (mean $\pm \mathrm{SD})$. - - - , fitted on raw data $(n=1354)$. $\cdots$, transposed from Fig. 6 for comparison. $B$ : time course of error between the 1st saccade $(\cdots)$ and the final orientation (- -) with 2 intermediate orientation errors. Time labels indicate the moment of sampling. $C$ : 3-dimensional representation of the position error as a function of SED and time.

ended $\sim 800 \mathrm{~ms}$ after the flash. Furthermore, this process compensated not only for SED but also for the saccadic gain error, which is present at the time of the first saccade. At the end of the orientation process, the total saccadic gain was 0.989 compared with 0.888 at $250 \mathrm{~ms}$ after the flash. For comparison, we provide in Fig. $8 B$ the mean and SD of the smooth eye velocity after the flash onset. In Fig. 8, smooth eye velocity traces are aligned on the flash onset and not on the gap onset, as this was the case in Fig. 3. Therefore in Fig. $8 B$, the variability of the smooth eye movement amplitude is partly due to the variability of the flash onset.

The open triangle symbols in Fig. 8, $A$ and $B$, correspond to individual data from the example in Fig. 9A. The coefficient of SED $(\delta)$ and the instantaneous smooth eye velocity were mea- sured after each saccade and follow the average time course of these parameters in Fig. 8, $A$ and $B$. The solid symbols in Fig. 8, $A$ and $B$, are associated with two other examples (Fig. 9, $B$ and $C$ ) and show that the time course of the coefficient of SED $[\delta(t)]$ was not influenced by the sequence of saccades. This can be observed when looking at the first saccade of $B$ and $C$ in Fig. 9. A comparison of $B$ with $A$ shows that SED compensation has already started for the first saccade of $B$, whereas this was not the case for the first saccade of $A$. This behavior is even more dramatic if we compare $C$ with $A$. For the first saccade in $C$, SED compensation is similar to that of the third saccade in $A$. This illustrates that only the time of saccadic execution determines the amount of SED compensation and not whether it is the first, second, or third saccade.

Dotted vertical lines in Fig. 8, $A$ and $B$, indicate different landmarks in the orientation process and the eye movement. These were obtained by determining when the measured variable fell $<10 \%$ of the maximum or rose $>10 \%$ of the minimum with respect to the total scale. We evaluated the beginning (dotted line 1) and the end (dotted line 3) of the orientation process at 363 and $835 \mathrm{~ms}$, respectively. With the same procedure, we measured the end of the smooth eye movement (dotted line 2) at $440 \mathrm{~ms}$ after the flash. Thus there was approximately the same delay of $400 \mathrm{~ms}$ between flash onset and the beginning of the compensation process and
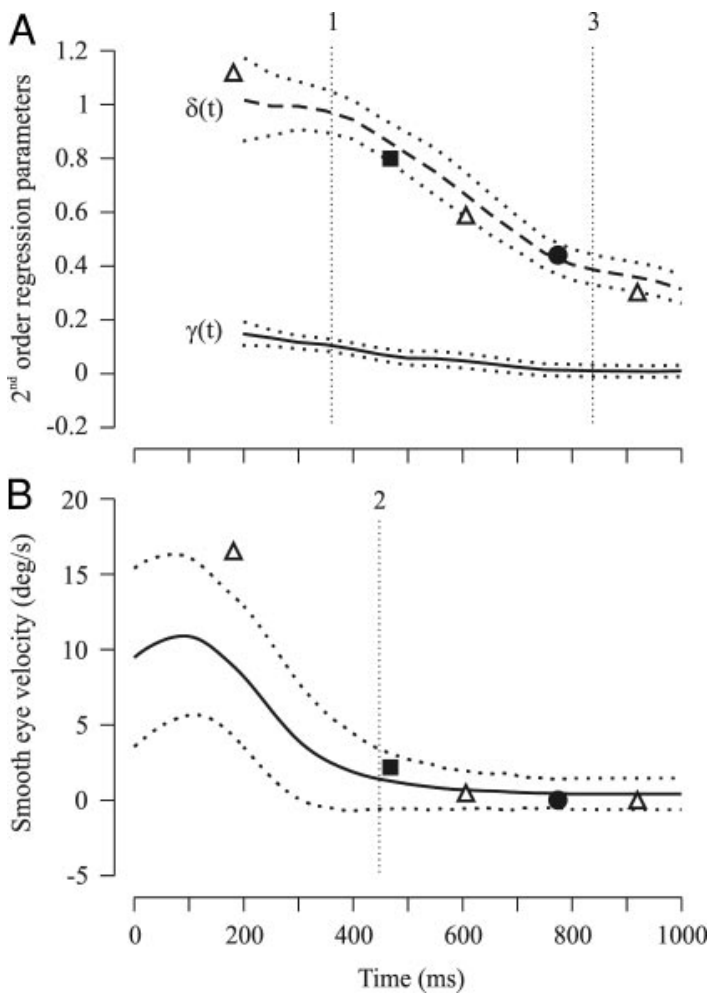

FIG. 8. Time course of the orientation process. A: 2nd-order regression coefficients of the error in time are represented (mean and 95\% confidence interval). Independent variables are the smooth eye displacement SED (coefficient $\delta(\mathrm{t})$, dashed line) and the position error at the moment of the flash $\mathrm{PE}_{\text {to }}$ (coefficient $\gamma(\mathrm{t})$, solid line). $B$ : mean (solid line) and SD (dotted lines) of the smooth eye velocity across all trials. Symbols in both panels refer to examples of Fig. 9 (see text). Vertical dotted lines represent the onset of the orientation process (1), the end of the smooth eye displacement (2) and the end of the orientation process (3). 

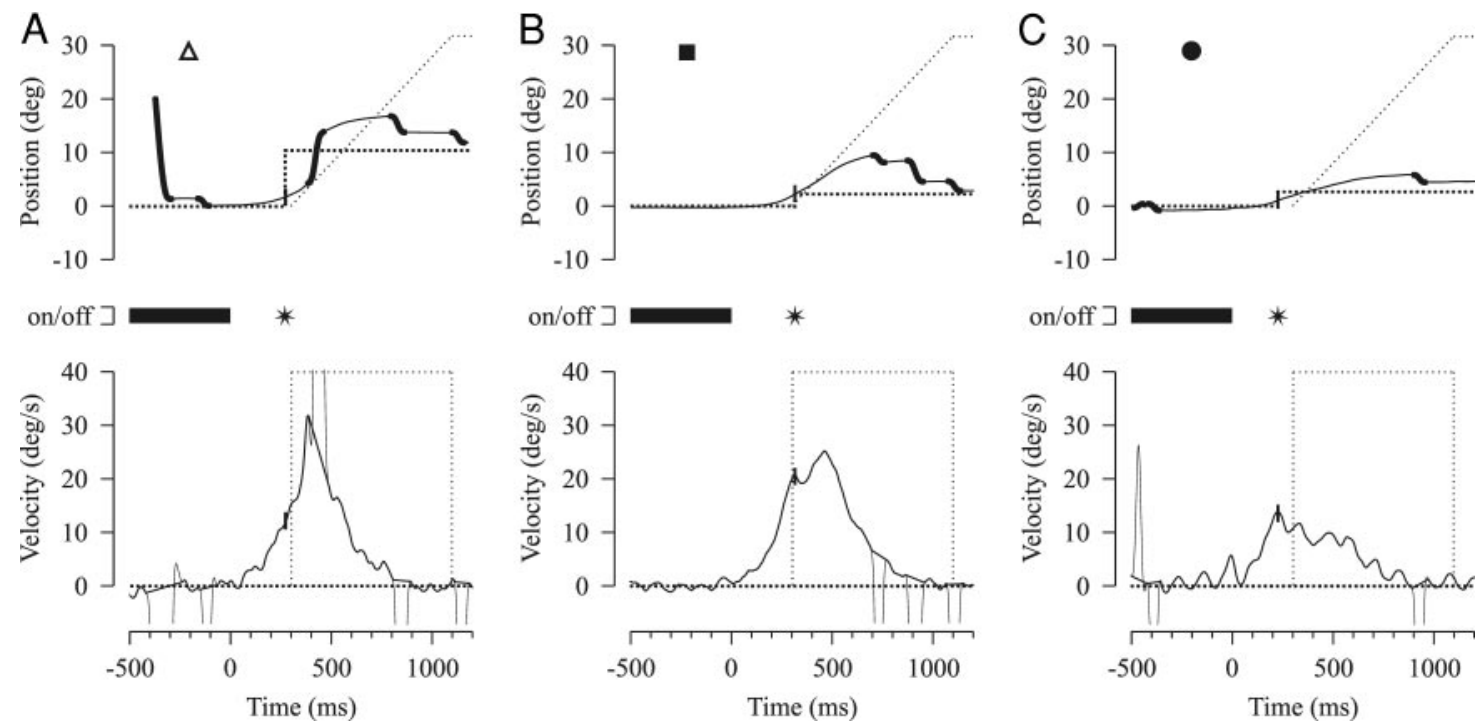

FIG. 9. Examples for the transient test condition to illustrate the time course of orientation in Fig. 8. The same conventions as in Fig. 2 are used. A: short 1st saccade latency $(106 \mathrm{~ms})$. B: delayed 1st saccade (latency $=412 \mathrm{~ms}$ ). $C$ : late 1 st saccade (latency $=716 \mathrm{~ms}$ ).

between the end of the smooth eye movement and the end of the orientation process.

In Fig. 10, $A$ and $B$, we tested the hypothesis that a constant delay model might explain the time course of the orientation process. This means that at a given instant in time $t$ subjects would compensate for the smooth eye movement accomplished up to time $t-T$. Thus the assumption that the compensation $\alpha$ is proportional to SED accumulated up to a delay $T$ before the measure of the position error $\mathrm{PE}(t)$ mathematically translates into the following expression

$$
\operatorname{PE}(t)=\operatorname{SED}(t)-\alpha(t) \cdot \operatorname{SED}(t-T)
$$

The first term, $\operatorname{SED}(t)$, describes the proportionality of the error to SED in the early orientation process. The second term is compensatory and accounts for the smooth eye displacement accumulated up to $t-T$. In comparison with $E q$. 5, we removed the term proportional to $\mathrm{PE}_{\text {to }}$ for this analysis because $E q$. 6 was simpler and the results were qualitatively the same. Figure $10 A$ shows the value of $\alpha(t)$ for different values of the delay $T$. The curve for $T=0 \mathrm{~ms}$ corresponds to the evolution of $\delta(t)$ in Fig. $8 A$. Interestingly, the value of $\alpha$ was a constant in time ( $\alpha \cong 0.7$ ) for a delay of $400 \mathrm{~ms}$. This is compatible with the hypothesis that the time course of the compensation process could be explained by a constant compensatory gain combined with a delayed signal of the smooth eye displacement. This hypothesis was confirmed by the analysis performed on each subject individually in Fig. 10B. For each subject, there was a specific time delay (ranging from 350 to $450 \mathrm{~ms}$ ) that yielded a constant compensatory gain $\alpha$ (ranging from 0.43 to 0.93 ).

\section{I S C U S S I O N}

In the absence of a smooth eye movement, saccades can be aimed toward the spatial location of a memorized or visual target (Becker and Jürgens 1979). In this study, we perturbed this condition by inducing a smooth anticipatory eye movement that participated in the gaze displacement. We found a linear addition of the smooth anticipatory and saccadic motor commands for all test conditions. Furthermore, in the sustained test condition, the saccadic system used a predictive component (based on the retinal slip) in catch-up saccade programming. In the transient test condition, the saccadic system did
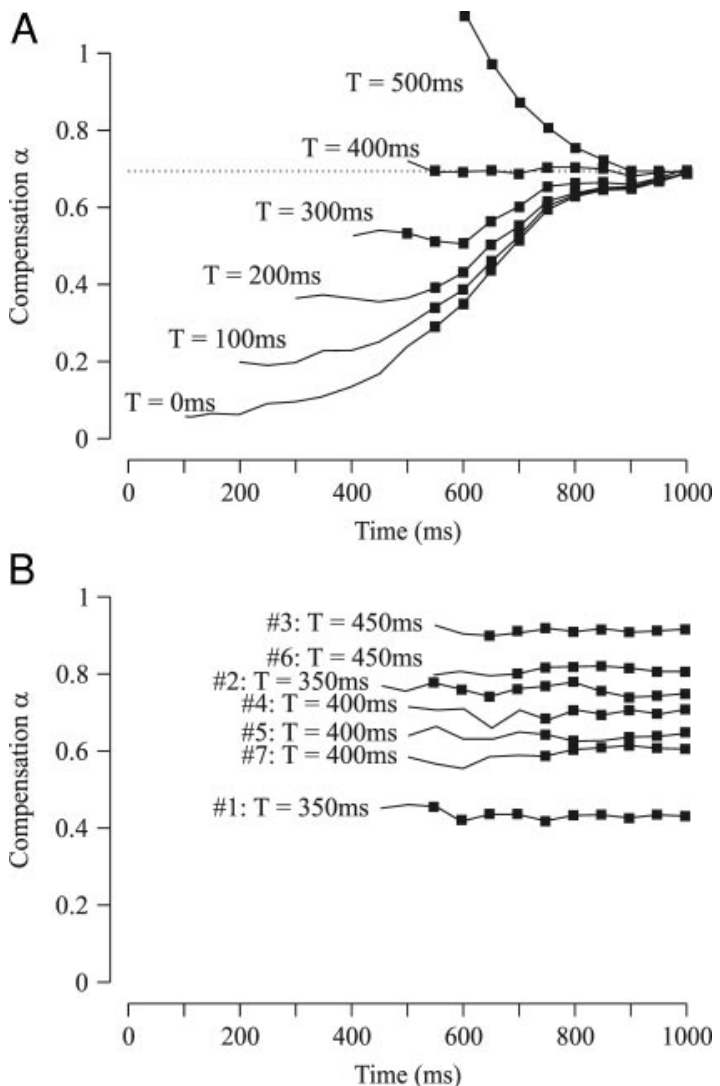

FIG. 10. Compensation $\alpha(t)$ of the smooth eye displacement SED (refer to $E q$. 6 in text). $A$ : - , the evolution of $\alpha$ in time for different delays $T$. Squares indicate when the compensation $\alpha(t)$ is significant $(P<0.01)$. For $T=400 \mathrm{~ms}, \alpha$ is approximately constant over time and equal to 0.69 . This corresponds exactly to the average contribution of SED to the final error $(1-\alpha$, see Figs. 7 and 8$)$. This is consistent with the hypothesis that compensation is a delayed process with a constant gain. $B$ : optimal compensation $\alpha$ of the smooth eye displacement SED for each subject. The optimal delay $T$ is also given. Numbers refer to the different subjects. 
not take into account the smooth anticipatory eye movements in the early stage of the orientation process toward the target. However, we provided strong evidence for a compensatory mechanism between both oculomotor subsystems later on in the orientation process. Thus the oculomotor system can rely on extraretinal information to control the coordination between its different components.

\section{Saccade properties}

The analysis of the main sequence relationship showed evidence that smooth anticipatory and saccadic drives are both operational and are linearly summated during the saccade. The same result was obtained previously for the smooth pursuit system (de Brouwer et al. 2002). This finding has two implications. First the smooth anticipatory motor system does not pause during saccades, and thus the smooth anticipatory component must be removed for the analysis of saccades executed during anticipation. Second, the saccadic system interacts in the same way with the smooth pursuit and smooth anticipatory systems. This finding is compatible with the hypothesis that these two smooth motor systems share common neural structures. This view is supported by several behavioral studies (Boman and Hotson 1988; Braun et al. 1996; Kao and Morrow 1994).

The saccadic latency histogram showed two main properties. First the minimum latency for saccades aimed at visual targets is very short $(\sim 80 \mathrm{~ms})$. The use of a gap in our paradigm and the fact that the eyes were moving at the appearance of the target might explain this behavior because both factors release active fixation (Krauzlis and Miles 1996a-c). Second, saccadic latency histograms in our experiment are well described by the LATER model (Reddi and Carpenter 2000). The recinormal function fitted our data significantly better than a normal distribution. Thus we showed that this model does not only apply to saccades following fixation but that it also describes the latencies of saccades triggered during smooth anticipatory eye movements in darkness.

\section{Programming of the first orienting saccade}

In the sustained test condition, the first orienting saccade was programmed using the position error and retinal slip sampled $100 \mathrm{~ms}$ before the saccade onset. The behavior was qualitatively the same as during smooth pursuit, and thus saccades were accurate (de Brouwer et al. 2002). However, the coefficient of RS that we found in Eq. 1 is smaller than in the study by de Brouwer et al. (2002), i.e., 0.059 versus 0.091 . It may be due partly to differences between subjects. Nevertheless, we believe that the main effect is due to the difference between the active pursuit paradigm of de Brouwer et al. (2002) and the anticipatory pursuit in darkness. In our paradigm, subjects had to reengage active pursuit after the target appearance, which might result in an underestimation of the retinal slip.

In the case of the transient test condition, the first saccade was programmed only on the basis of the position error at the moment of the flash, which was the only retinal information available to the oculomotor system in this condition. In several previous studies, subjects had to orient gaze toward a target that was briefly flashed after the disappearance of a smooth pursuit target (Gellman and Fletcher 1992; McKenzie and Lisberger 1986; Schlag et al. 1990). These studies are compatible with our finding that first orienting saccades only account for the position error at the moment of the flash. However, these studies only reported data on the first orienting saccade and did not give any indication about the orientation process going on afterward.

\section{Time course of the orientation process}

The presence of multiple orientation saccades in our paradigm revealed a compensatory mechanism that accounted for the smooth eye displacement. Compensation started $\sim 400 \mathrm{~ms}$ after the flash and lasted until $\sim 400 \mathrm{~ms}$ after the end of the smooth eye movement. This process is compatible with the hypothesis of a delayed compensation mechanism. The delay of $400 \mathrm{~ms}$ explains the time course of the compensation process and the apparent evolution of the compensatory gain in Fig. 8. This hypothesis has been confirmed independently in each subject, with a fairly constant delay $(400 \pm 50 \mathrm{~ms})$ associated with a subject-specific constant compensation gain.

What is the origin of this 400-ms delay? Because the compensation is only apparent after orienting saccades, this delay clearly includes several components. First it includes the time necessary to make the decision to trigger a saccade and to program this saccade (estimation $\cong 75 \mathrm{~ms}$ ). Second, there is the duration for the execution of the saccade (mean $\cong 75 \mathrm{~ms}$ in our data). The last component $(250 \mathrm{~ms}=400-150 \mathrm{~ms})$ reflects some internal delay between the execution of the smooth eye movement and the time when an efferent copy of the smooth motor command can be integrated (to provide SED) and used by the saccadic system.

In our analysis, we found an overall compensation gain of 0.7 , which is not perfect. This partial compensation could be related to the fact that targets flashed during a movement may be perceptually mislocalized (see Schlag and Schlag-Rey 2002 for a review). This perceptual mislocalization, which is called the flash-lag effect, may influence the compensation gain we obtained.

\section{Proposed model}

During orientation toward visual targets, catch-up saccades use retinal slip information to interact with the smooth pursuit system. Here, we disrupted the ability of the saccadic system to access retinal information about the relative target displacement. Nevertheless, the saccadic system could account for the smooth eye displacement, although with a 400-ms delay. On the one hand, this delayed mechanism suggests that the saccadic system has to rely on an efference copy signal of the smooth motor command. We consider that an efference copy is the only available signal because proprioception is unlikely to play a role in ocular orientation (Lewis et al. 2001). On the other hand, the length of the delay $(400 \mathrm{~ms})$ might reflect the implication of several sub-cortical and cortical areas in this pathway.

We propose a model that may account for the observed compensation mechanism (Fig. 11). This model is composed of three distinct parts: the smooth system (left), an integrator of the smooth motor command (middle), and the saccadic system $(r i g h t)$. The gap onset acts as a cue for the smooth system to 


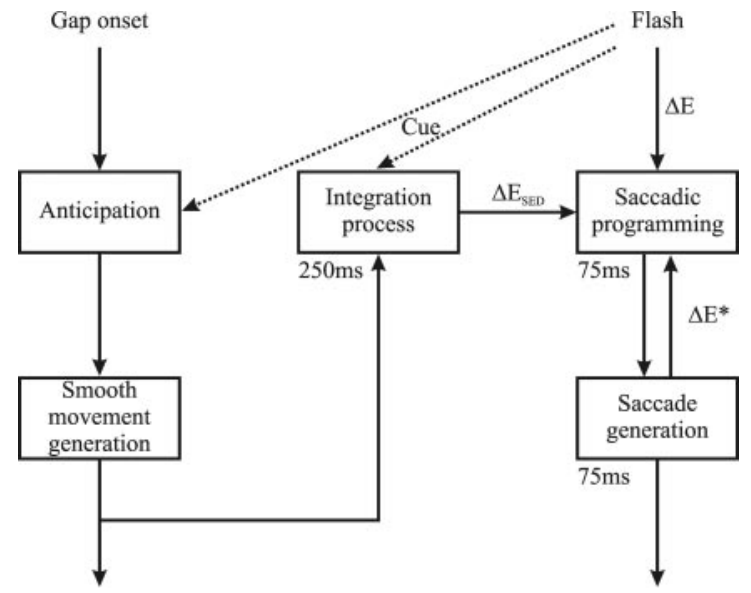

FIG. 11. Proposed model for monitoring smooth eye displacements in the absence of visual feedback. Left: smooth system. Middle: integration of the smooth motor command. Right: saccadic system. Time labels indicate the different hypothesized delays in the signal processing stage. In our paradigm, the gap onset instructed the smooth system to start anticipatory pursuit. In the transient test condition, the flash acted as a cue to stop the anticipatory drive and to reset the integrator of the smooth motor command (to obtain SED). A retinal position error $\Delta E=\mathrm{PE}_{\mathrm{to}}$ is used to program the first orienting saccade. Afterward, the integrator sends delayed information about the smooth eye displacement $\Delta E_{\mathrm{SED}}$ to the saccadic system. This information is used by the saccadic system to compensate for the smooth eye displacement. For more details, see text.

generate an anticipatory motor command that is sent to the smooth movement generator to anticipate the expected ramp target. During the test trials, either a sustained or a flashed target appears. Because the orientation to sustained targets relies on known mechanisms (de Brouwer et al. 2002), we will only consider the case of the flashed targets in the proposed model.

We hypothesize that the flash influences the smooth and saccadic systems. On the one hand, the flash occurrence is a cue to the smooth motor system to stop the anticipatory eye movement. At the same time this cue resets the integrator of the smooth motor command, which provides SED to the saccadic system. On the other hand, the location of the flash determines a goal $\Delta E$ for the saccadic system. A first shortlatency orienting saccade is executed based on the retinal error information provided by the flash. If the saccade is correct, the efference copy of the saccadic motor command $\Delta E^{*}$ is equal to the initial position error $\Delta E$ and the goal is achieved. But meanwhile the eyes have been perturbed by the smooth system. The integrator of the smooth motor command sends a delayed (250 ms) smooth eye displacement signal $\Delta E_{\mathrm{SED}}$ to the saccadic system. Based on this information a new saccade is programmed $(75 \mathrm{~ms})$ and executed $(75 \mathrm{~ms})$. This process is repeated until the end of the smooth eye movement. Again because it involves such long delays, this pathway is only predominant if no retinal information is available.

We will try to propose a hypothesis about the underlying neural correlates that could support our model. Details about the smooth pursuit and saccade generators will not be discussed here (see for example Krauzlis and Stone 1999 for a review). Here, we concentrate on the pathway integrating the smooth motor command and programming the compensatory saccades. As we already mentioned, the 400 -ms delay suggests that the integration of the smooth motor command takes place in the cerebral cortex. The internal representation of the smooth eye displacement $\Delta E_{\mathrm{SED}}$ could be used to update the memorized spatial representation of the flashed target.

We propose that the parietal cortex might play a relay role between the smooth pursuit and saccadic systems because areas implied in both types of eye movements project to this brain region. Furthermore, the parietal cortex is strongly implied in processing extraretinal signals (Tobler et al. 2001) and is important for self-movement integration (Duhamel et al. 1992; Heide et al. 1995). Lateral intraparietal region (LIP) and area 7 a receive information about the saccadic commands to encode the location of the visual stimulus in spatial coordinates (Andersen et al. 1985; Bremmer et al. 1997). In addition, LIP neurons discharge prior to saccades and remain active while remembering a desired target location (Barash et al. 1991; Paré and Wurtz 1997) and lesions of the posterior parietal cortex impair the ability to make memory-guided saccades (PierrotDeseilligny et al. 1991).

Following our hypothesis not only inputs from the saccadic system could update the internal target representation in the parietal cortex but there might also be a contribution from the smooth pursuit system accounting for the smooth eye displacement. In fact, the smooth pursuit system communicates bilaterally with the posterior parietal cortex (area 7a) via the medial superior temporal (MST) area (Tusa and Ungerleider 1988). Neurons in MST carry information about the smooth eye movements (Newsome et al. 1988) that might come from an efference copy of the smooth motor command (Leigh and Zee 1999). Thus smooth movement information could update the internal representation of targets in space, and saccades could be triggered whenever the parietal cortex communicates information about a smooth eye displacement to the saccadic system.

\section{Conclusion}

In this paper, we studied the interaction between smooth anticipatory eye movements and saccades. Saccades triggered during smooth anticipation toward a sustained visual target are programmed using the available retinal input, as is the case during sustained smooth pursuit. If flashed targets are presented, no retinal information about the movement is available to program adequate saccades. However, saccades can correct for the smooth eye displacement that took place some time before and this process has been estimated to take $\sim 400 \mathrm{~ms}$. Thus we believe that the saccadic system has access to an efference copy of the smooth motor command to monitor the smooth eye displacement.

This work was supported by the Fonds National de la Recherche Scientifique; the Fondation pour la Recherche Scientifique Médicale; the Belgian program on inter-university poles of attraction initiated by the Belgian state, Prime Minister's office for Science, Technology and Culture (SSTC); and internal research grant (Fonds Spéciaux de Recherche) of the Université Catholique de Louvain.

\section{REFERENCES}

Andersen RA, Essick GK, and Siegel RM. Encoding of spatial location by posterior parietal neurons. Science 230: 456-458, 1985.

Aslin RN and Shea SL. The amplitude and angle of saccades to double-step target displacements. Vision Res 27: 1925-1942, 1987.

Barash S, Bracewell RM, Fogassi L, Gnadt JW, and Andersen RA. Saccade-related activity in the lateral intraparietal area. II. Spatial properties. J Neurophysiol 66: 1109-1124, 1991. 
Barnes GR and Asselman PT. The mechanism of prediction in human smooth pursuit eye movements. J Physiol 439: 439-461, 1991.

Becker W and Jürgens R. An analysis of the saccadic system by means of double step stimuli. Vision Res 19: 967-983, 1979.

Boman DK and Hotson JR. Stimulus conditions that enhance anticipatory slow eye movements. Vision Res 28: 1157-1165, 1988.

Braun DI, Boman DK, and Hotson JR. Anticipatory smooth eye movements and predictive pursuit after unilateral lesions in human brain. Exp Brain Res 110: 111-116, 1996.

Bremmer F, Distler C, and Hoffmann KP. Eye position effects in monkey cortex. II. Pursuit- and fixation-related activity in posterior parietal areas LIP and 7A. J Neurophysiol 77: 962-977, 1997.

Collewijn H, Van Der Mark F, and Jansen TC. Precise recording of human eye movements. Vision Res 15: 447-450, 1975.

Dassonville P, Schlag J, and Schlag-Rey M. The frontal eye field provides the goal of saccadic eye movement. Exp Brain Res 89: 300-310, 1992.

De Brouwer S, Missal M, Barnes GR, and Lefèvre P. Quantitative analysis of catch-up saccades during sustained pursuit. J Neurophysiol 87: 1772$1780,2002$.

De Brouwer S, Missal M, and Lefèvre P. Role of retinal slip in the prediction of target motion during smooth and saccadic pursuit. J Neurophysiol 86 $550-558,2001$

Dominey PF, Schlag J, Schlag-Rey M, and Arbib MA. Colliding saccades evoked by frontal eye field stimulation: artifact or evidence for an oculomotor compensatory mechanism underlying double-step saccades? Biol Cybern 76: 41-52, 1997.

Duhamel JR, Goldberg ME, Fitzgibbon EJ, Sirigu A, and Grafman J. Saccadic dysmetria in a patient with a right frontoparietal lesion. The importance of corollary discharge for accurate spatial behavior. Brain 115: 1387-1402, 1992.

Gellman RS and Fletcher WA. Eye position signals in human saccadic processing. Exp Brain Res 89: 425-434, 1992.

Goldman-Rakic PS. Circuitry of primate prefrontal cortex and regulation of behavior by representational memory. In: Handbook of Physiology. The Nervous System. Higher Functions of the Brain. Bethesda, MD: Am. Physiol. Soc., Bethesda, 1987, sect. 1, vol. 5, p. 373-417.

Goossens HH and Van Opstal AJ. Local feedback signals are not distorted by prior eye movements: evidence from visually evoked double saccades. J Neurophysiol 78: 533-538, 1997.

Hallett PE and Lightstone AD. Saccadic eye movements to flashed targets. Vision Res 16: 107-114, 1976a.

Hallett PE and Lightstone AD. Saccadic eye movements towards stimuli triggered by prior saccades. Vision Res 16: 99-106, 1976b.

Heide W, Blankenburg M, Zimmermann E, and Kompf D. Cortical control of double-step saccades: implications for spatial orientation. Ann Neurol 38: 739-748, 1995 .

Heywood S and Churcher J. Saccades to step-ramp stimuli. Vision Res 21: 479-490, 1981.

Kao GW and Morrow MJ. The relationship of anticipatory smooth eye movement to smooth pursuit initiation. Vision Res 34: 3027-3036, 1994.

Kowler E, Martins AJ, and Pavel M. The effect of expectations on slow oculomotor control. IV. Anticipatory smooth eye movements depend on prior target motions. Vision Res 24: 197-210, 1984

Krauzlis RJ and Miles FA. Decreases in the latency of smooth pursuit and saccadic eye movements produced by the "gap paradigm" in the monkey. Vision Res 36: 1973-1985, 1996a.

Krauzlis RJ and Miles FA. Initiation of saccades during fixation or pursuit: evidence in humans for a single mechanism. J Neurophysiol 76: 4175-4719, 1996b.

Krauzlis RJ and Miles FA. Release of fixation for pursuit and saccades in humans: evidence for shared inputs acting on different neural substrates. J Neurophysiol 76: 2822-2833, 1996c
Krauzlis RJ and Miles FA. Role of the oculomotor vermis in generating pursuit and saccades: effects of microstimulation. J Neurophysiol 80: 20462062, 1998.

Krauzlis RJ and Stone LS. Tracking with the mind's eye. Trends Neurosci 22: $544-550,1999$.

Leigh JR and Zee DS. The Neurology of Eye Movements (3rd ed.). New York: Oxford, 1999.

Lewis RF, Zee DS, Hayman MR, and Tamargo RJ. Oculomotor function in the rhesus monkey after deafferentation of the extraocular muscles. Exp Brain Res 141: 349-358, 2001

Mays LE and Sparks DL. Saccades are spatially, not retinocentrically, coded. Science 208: 1163-1165, 1980.

McKenzie A and Lisberger SG. Properties of signals that determine the amplitude and direction of saccadic eye movements in monkeys. $J$ Neurophysiol 56: 196-207, 1986.

Missal M, De Brouwer S, Lefèvre P, and Olivier E. Activity of mesencephalic vertical burst neurons during saccades and smooth pursuit. $J$ Neurophysiol 83: 2080-2092, 2000.

Missal M and Heinen SJ. Facilitation of smooth pursuit initiation by electrical stimulation in the supplementary eye fields. J Neurophysiol 86: 2413-2425, 2001.

Missal $\mathbf{M}$ and Keller EL. Common inhibitory mechanism for saccades and smooth-pursuit eye movements. J Neurophysiol 88: 1880-1892, 2002.

Morrow MJ and Lamb NL. Effects of fixation target timing on smoothpursuit initiation. Exp Brain Res 111: 262-270, 1996.

Mushiake H, Fujii N, and Tanji J. Microstimulation of the lateral wall of the intraparietal sulcus compared with the frontal eye field during oculomotor tasks. J Neurophysiol 81: 1443-1448, 1999.

Newsome WT, Wurtz RH, and Komatsu H. Relation of cortical areas MT and MST to pursuit eye movements. II. Differentiation of retinal from extraretinal inputs. J Neurophysiol 60: 604-620, 1988.

Paré M and Wurtz RH. Monkey posterior parietal cortex neurons antidromically activated from superior colliculus. J Neurophysiol 78: 3493-3497, 1997.

Pierrot-Deseilligny C, Rivaud S, Gaymard B, and Agid Y. Cortical control of memory-guided saccades in man. Exp Brain Res 83: 607-617, 1991.

Reddi BA and Carpenter RH. The influence of urgency on decision time. Nat Neurosci 3: 827-828, 2000

Robinson DA. A method of mesuring eye movement using a scleral search coil in a magnetic field. IEEE, Trans Biomed Eng BME 10: 137-145, 1963.

Schlag J and Schlag-Rey M. Colliding saccades may reveal the secret of their marching orders. Trends Neurosci 13: 410-415, 1990.

Schlag J and Schlag-Rey M. Through the eye, slowly: delays and localization errors in the visual system. Nat Rev Neurosci 3: 191-200, 2002.

Schlag J, Schlag-Rey M, and Dassonville P. Interactions between natural and electrically evoked saccades. II. At what time is eye position sampled as a reference for the localization of a target? Exp Brain Res 76: 548-558, 1989.

Schlag J, Schlag-Rey M, and Dassonville P. Saccades can be aimed at the spatial location of targets flashed during pursuit. J Neurophysiol 64: 575$581,1990$.

Schlag-Rey M, Schlag J, and Shook B. Interactions between natural and electrically evoked saccades. I. Differences between sites carrying retinal error and motor error signals in monkey superior colliculus. Exp Brain Res 76: 537-547, 1989.

Tian J, Schlag J, and Schlag-Rey M. Testing quasi-visual neurons in the monkey's frontal eye field with the triple-step paradigm. Exp Brain Res 130: 433-440, 2000.

Tobler PN, Felblinger J, Burki M, Nirkko AC, Ozdoba C, and Muri RM. Functional organization of the saccadic reference system processing extraretinal signals in humans. Vision Res 41: 1351-1358, 2001.

Tusa RJ and Ungerleider LG. Fiber pathways of cortical areas mediating smooth pursuit eye movements in monkeys. Ann Neurol 23: 174-183, 1988. 